\section{Fatores associados à anemia em crianças e adolescentes de escolas públicas de Salvador, Bahia, Brasil}

\author{
Factors associated with anemia in children and \\ adolescents in public schools in Salvador, Bahia \\ State, Brazil
}

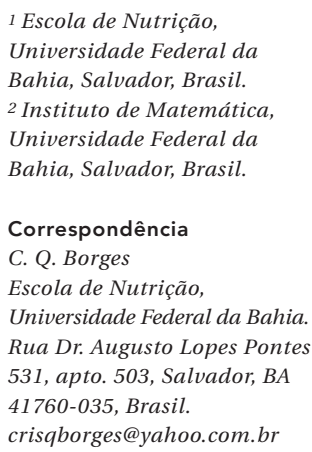

\begin{abstract}
This study aimed to assess factors associated with anemia in schoolchildren. All subjects ( $N=1,013$; age 7-14 yrs.) were students enrolled in the public school system in Salvador, Bahia State, Brazil. Blood tests were done to determine hemoglobin levels, anthropometric data were collected, and a 24-hour food recall was performed. The study also gathered socioeconomic, environmental, and household data, as well as information on care by the mother. Anemia was detected in $24.5 \%$ of subjects, with mean hemoglobin of $12.68 \mathrm{~g} / \mathrm{dL}$ (range $7.7 \mathrm{~g}-16.8 \mathrm{~g} / \mathrm{dL}$ ). Anemia was significantly associated with inadequate intake of bioavailable iron $\left(\mathrm{OR}_{50-99.9 \%}\right.$ adequacy $=1.57$; 95\% CI: 1.07 2.29; $p=0.020$ and $O R_{<50 \% \text { adequacy }}=1.68 ; 95 \%$ CI: $1.10-2.56 ; p=0.016)$ and family income below 1 minimum wage $(\mathrm{OR}=1,42$; 95\%CI: 1.03-1.96; $p$ $=0.035$ ). Anemia prevalence in children and adolescents was high, especially among the poorest subjects and those with low intake of bioavailable iron.
\end{abstract}

Anemia; Food Consumption; Child; Adolescent

\author{
Cristiane Queiroz Borges 1 \\ Rita de Cássia Ribeiro Silva ${ }^{1}$ \\ Ana Marlúcia Oliveira Assis ${ }^{1}$ \\ Elizabete de Jesus Pinto 1 \\ Rosemeire Leovigildo Fiaccone 2 \\ Sandra Maria Conceição Pinheiro ${ }^{1}$
}

\section{Introdução}

A anemia por deficiência de ferro é considerada um dos problemas nutricionais de maior relevância, acometendo cerca de 30\% da população mundial, notadamente em regiões mais pobres 1 . Segundo a Organização Mundial da Saúde (OMS), anemia nutricional é a condição na qual o conteúdo de hemoglobina do sangue está abaixo dos valores considerados normais, em conseqüência da carência de um ou mais nutrientes essenciais, necessários à formação da hemoglobina ${ }^{2}$. Embora vários nutrientes possam estar envolvidos na instalação de um quadro de anemia, a deficiência de ferro tem sido reconhecida como a causa mais importante, sendo, em nosso meio, responsável por $90 \%$ das anemias nutricionais 2 .

A anemia constitui um importante problema de saúde, quer pela elevada prevalência, quer pelos seus efeitos deletérios sobre a saúde humana. Em crianças e adolescentes tem sido identificado efeito negativo da anemia sobre o crescimento pondero-estatural ${ }^{3}$, o desenvolvimento psicomotor e a aprendizagem 4,5 . Essas conseqüências variam de acordo com a gravidade e duração da anemia e podem persistir mesmo após a correção da deficiência 6 .

A anemia é o resultado do desequilíbrio entre a quantidade do mineral biologicamente disponível e a necessidade orgânica. A alta prevalência dessa deficiência nutricional na criança e no adolescente é o reflexo do baixo consumo de alimen- 
tos fonte de ferro biodisponível e do aumento da demanda orgânica do mineral, ocasionado pela expansão dos tecidos e da massa celular vermelha ${ }^{7}$. Além disso, de patologias, como as doenças parasitárias, em especial as infecções helmínticas, determinadas pelas precárias condições de saúde e sanitárias em que vivem as crianças e seus familiares. Nesse contexto, deve-se considerar que as condições de saneamento e moradia, além das práticas de consumo alimentar sofrem influência do padrão de vida familiar, que, em última instância, é determinado pela situação social e econômica da população.

Alguns fatores associados à anemia estão bem documentados na infância e em gestantes. No entanto, existem lacunas importantes no conhecimento desse problema de saúde, quando o grupo alvo é representado por crianças em idade escolar e adolescentes. Assim, o presente trabalho foi planejado para estimar a prevalência da anemia e identificar os fatores associados a essa deficiência nutricional.

\section{Métodos e técnicas}

Trata-se de um estudo desenvolvido no bojo de uma investigação mais ampla intitulada Situação Nutricional, Hábitos Alimentares e Desenvolvimento Cognitivo de Estudantes do Ensino Fundamental das Escolas Públicas de Salvador/BA, realizada pela Escola de Nutrição da Universidade Federal da Bahia. Participaram do presente estudo 1.013 alunos de ambos os sexos, com idades de 7 a 14 anos, e identificados pelo uso das bases de dados da matrícula realizada em 2006, disponibilizadas pela Secretaria de Educação e Cultura do Município e pela Secretaria de Educação do Estado da Bahia.

O processo de amostragem envolveu um desenho complexo, valendo-se da estratificação das escolas em dois níveis (estadual e municipal), seguido pelo procedimento de amostragem por conglomerado em três estágios descrito a seguir: o primeiro estágio representado pelos distritos sanitários, o segundo pelas escolas e finalmente, o último, pelos alunos. Utilizando as informações do banco de matrícula disponibilizado pelos órgãos citados anteriormente, em que foram constatadas 217 escolas estaduais (com 103.924 alunos) e 354 escolas municipais (com 114.464) distribuídas nos 12 distritos sanitários que compõem o Município de Salvador, Bahia, Brasil, foram selecionados aleatoriamente seis distritos para integrar a referida amostra. Optouse por esse número de distrito por questões logísticas de campo. Dos seis distritos sorteados, foram computadas 117 escolas estaduais e 173 municipais. As escolas estaduais comportavam 58.059 alunos e as municipais 56.555. Com um nível de $95 \%$ de confiança, uma precisão absoluta de $3 \%$ e adotando-se a prevalência de anemia de $35 \%$ identificada por Brito et al. ${ }^{8}$, calculou-se um amostra de 963 alunos. Considerando a possibilidade de perda de $25 \%$, a amostra final foi estimada em 1.201 escolares, distribuída em 58 escolas municipais e 27 estaduais Para atender ao número amostral previamente definido, verificou-se a necessidade de selecionar em média dez alunos em cada unidade municipal e 23 nas unidades estaduais.

Do total de escolares inicialmente selecionados, registrou-se a perda de 188 (15,65\%) deles. Essas perdas ocorreram devido à recusa $(0,67 \%)$ e à mudança da criança para outra cidade; ou transferência para outra escola (14,98\%). Assim, a amostra efetivamente estudada constituiu-se de 1.013 indivíduos.

A coleta de sangue para dosagem de hemoglobina foi feita em campo, por puntura digital, aplicando-se o método da cianometa-hemoglobina. Para a realização dessa coleta, utilizou-se o hemoglobinômetro portátil HemoCue (HemoCue Inc., Laguna Hills, Estados Unidos), sendo o aparelho calibrado diariamente, conforme as especificações do fabricante. $\mathrm{O}$ aparelho tem sido utilizado em estudos populacionais devido à maior praticidade e menor característica invasiva. Foi também previamente demonstrado ser preciso, quando comparado a outros procedimentos laboratoriais padrões, possuir elevada sensibilidade e especificidade, sendo não confiável apenas quando requerida a estimativa de níveis individuais 9. A anemia foi definida mediante os pontos de corte propostos por Unite for Children/United Nations University/World Health Organization/Micronutrient Initiative 10: $\mathrm{Hb}<$ 11,5g/dL para indivíduos na faixa etária de 7 a 11 anos e $\mathrm{Hb}<12 \mathrm{~g} / \mathrm{dL}$ entre os de 12 e 14 anos.

As informações sobre o consumo alimentar de todos os alunos que participaram do presente estudo foram coletadas pelo método de inquérito recordatório de 24 horas, em dias típicos dessa população. Um álbum, contendo os desenhos de porções diferenciados dos alimentos e utensílios, foi utilizado para minimizar o viés de memória. O cálculo da composição centesimal de macro (carboidratos, proteínas e lipídios) e micro-nutrientes (ferro total e vitamina C) da dieta foi realizado por meio do programa Virtual Nutri (Departamento de Nutrição, Faculdade de Saúde Pública, Universidade de São Paulo, São Paulo, Brasil). Para avaliação da adequação de consumo de calorias, proteína e ferro das dietas foram considerados os valores médios de referência, segundo sexo e idade, preconizados pela 
Food and Agriculture Organization/World Health Organization/United Nations University 11,12.

O ferro biodisponível foi quantificado por meio da equação desenvolvida por Monsen \& Balintfy 13, a qual prediz o potencial de absorção desse nutriente em cada uma das refeições que compôs a alimentação diária. Para essa quantificação levou-se em consideração a composição de cada refeição com relação a ferro total, ferro heme, ferro não-heme e quantidade de ácido ascórbico e de carnes.

O peso foi obtido utilizando-se balança portátil microeletrônica, marca Marte, modelo PP20050 (Marte Balanças e Aparelhos de Precisão Ltda., São Paulo, Brasil), com capacidade para 199,95kg e precisão de $50 \mathrm{~g}$. Para determinar a altura, foi usado o estadiômetro (Leicester Height Measure, Londres, Inglaterra), graduado em décimos de centímetros. Foram realizadas duas medidas do peso corpóreo e da altura por dois antropometristas independentes, admitindo-se variação mínima de 1,00mm para medida de altura e 0,1kg para medida de peso. Quando os dois resultados ultrapassavam as variações mínimas permitidas, uma terceira medida foi aferida. A média entre as medidas mais próximas representou a média final. As medições foram realizadas conforme técnica preconizada por Lohman et al. 14. O índice de massa corporal (IMC) determinado pela razão entre o peso em quilogramas $(\mathrm{kg})$ e a altura em metros ao quadrado $\left(\mathrm{m}^{2}\right)$. Para avaliar o estado antropométrico, foram utilizadas como padrão de referência as tabelas de percentis do Centers for Disease Control and Prevention (CDC) 15 e a classificação foi realizada com base nos pontos de corte preconizados pela OMS 16 .

Utilizaram dados acerca das características do domicílio (condições de posse do domicílio, tipo de construção, material predominante de piso, material predominante na cobertura e parte do domicílio, número de habitantes por dormitórios etc.) e de saneamento básico (abastecimento de água, coleta de lixo, esgotamento sanitário) para a construção de um índice adaptado do modelo proposto por Issler \& Giugliani ${ }^{17}$. A cada situação foi atribuída uma pontuação, tendo a mais favorável recebido o valor 0 , e a mais desfavorável, a pontuação 1 . O somatório desses valores caracterizou o indicador das condições de moradia, o qual foi classificado em dois estratos: adequado (escore $\leq 4$ ) e inadequado (escore $>4$ ).

Para a comparação das prevalências de anemia segundo as características da população estudada, empregou-se o teste do qui-quadrado $\left(\chi^{2}\right)$. A magnitude da associação entre os fatores de risco e a ocorrência de anemia foi expressa em odds ratio (OR) e respectivos intervalos de 95\% de confiança (IC95\%). O processo de modelagem baseou-se em estratégia ordenada nas etapas reveladas a seguir. Primeiro, foram selecionadas as variáveis que apresentaram um valor de $\mathrm{p} \leq 0,20$ na análise univariada, conforme critério sugerido por Hosmer \& Lemeshow ${ }^{18}$. Posteriormente, realizou-se a análise multivariada empregando a técnica de regressão logística, embasada no modelo teórico definido a priori, respeitando-se a hierarquia existente entre os níveis de determinação da anemia, conforme apresenta a Figura 1. Nesse modelo, foi enfatizado o determinismo sócio-econômico-demográfico e alimentar como o principal fator desencadeador, direto ou indireto, da sucessão de eventos que culminaram com a anemia. Permaneceram, no modelo, as variáveis que mostraram níveis de significância estatística menor do que $5 \%$.

$\mathrm{Na}$ dimensão estrutural, integraram-se ao modelo as variáveis relacionadas às condições sócio-econômicas e de moradia, representadas pela renda ( $\geq 1$ salário mínimo; $<1$ salário mínimo) e indicador das condições de moradia (adequado; inadequado). Na dimensão das causas intermediárias, foram incorporadas as variáveis relacionadas ao cuidado materno dispensado à criança: escolaridade materna (até a $4 \underline{a}$ série; $5 \underline{a}$ a 8a série; 2 o grau ou mais); número de crianças no domicílio com menos do que cinco anos de idade ( $\leq 2$ crianças; $\geq 3$ crianças); e número de habitantes por dormitório ( $\leq 2$ habitantes/dormitório; $\geq$ 3 habitantes/dormitório). Na dimensão das causas proximais foram considerados dois blocos: aquele representado por variáveis relacionadas diretamente à criança e ao adolescente, sexo (masculino; feminino); idade (7 a 9,99 anos; 10 a 14,99 anos); e estado antropométrico (eutrofia; magreza; sobrepeso/obesidade); e o bloco representado por variáveis relacionadas ao consumo alimentar: adequação de caloria $(<50 \%$ de adequação; de $50 \%$ a $99,9 \%$ de adequação; $\geq 100 \%$ de adequação), adequação de proteína $(<50 \%$ de adequação; de 50\% a 99,9\% de adequação; $\geq$ $100 \%$ de adequação), adequação de ferro total (<50\% de adequação; de $50 \%$ a $99,9 \%$ de adequação; $\geq 100 \%$ de adequação) e adequação de ferro biodisponível (<50\% de adequação; de 50\% a 99,9\% de adequação; $\geq 100 \%$ de adequação).

As análises estatísticas foram corrigidas pelo delineamento complexo da amostra, por meio da utilização do conjunto de comandos SVY do Stata versão 9.0 (Stata Corp., College Station, Estados Unidos).

De acordo com as normas propostas para pesquisas envolvendo seres humanos, o protocolo de estudo foi submetido ao Comitê de Ética do Instituto de Saúde Coletiva, da Universidade Federal da Bahia, o qual apreciou e emitiu parecer favorável sobre a pertinência ética da in- 


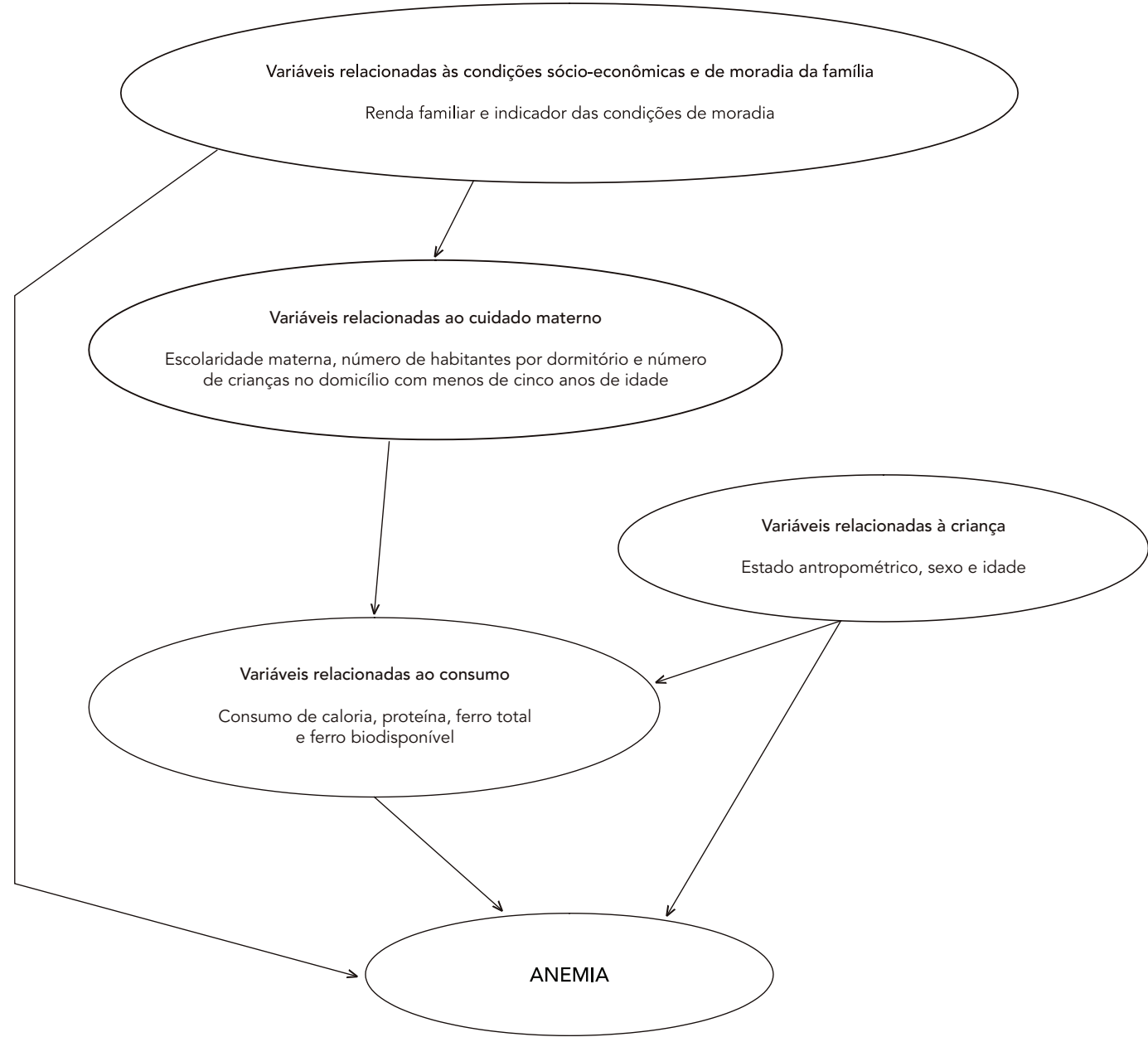

vestigação. Os alunos que apresentarem algum problema nutricional, diagnosticado neste estudo, foram encaminhados com orientação para acompanhamento em unidades da rede do Sistema Único de Saúde (SUS) ou outro serviço de saúde de sua preferência.

\section{Resultados}

Compuseram a população deste estudo 1.013 crianças e adolescentes de ambos os sexos, com idade de 7 a 14 anos. Foi detectada uma prevalência de anemia de $24,5 \%$ entre os participantes envolvidos no estudo, sendo $1 \%$ deles portador de anemia na forma grave $(\mathrm{Hb}<9,5 \mathrm{~g} / \mathrm{dL})$. O valor médio dos níveis de hemoglobina nessa população foi de 12,68g/dL (erro padrão - EP = 0,41), com concentração mínima de $7,7 \mathrm{~g} / \mathrm{dL}$ e máxima de $16,8 \mathrm{~g} / \mathrm{dL}$.

Dentre as variáveis de exposição que caracterizam as condições sócio-econômicas, demográficas e do consumo alimentar dos participantes, evidenciou-se maior número de anêmicos entre aqueles oriundos de famílias que recebiam menos do que um salário mínimo (Tabela 1) e com adequação de consumo de ferro total menor do que 50\% (Tabela 2); para as demais variáveis, não se observou distribuição diferenciada entre os anêmicos, conforme verificado nas Tabelas 1, 2 e 3. O resultado da análise univariada indicou que a renda familiar mensal menor do que um salá- 
rio mínimo se associou significantemente com a anemia, diferentemente do que ocorreu quando se avaliou a relação entre a renda maior que o salário mínimo e a anemia (OR = 1,37; IC95\%: 1,00-1,90) (Tabela1).
$\mathrm{Na}$ análise de regressão logística múltipla, embasada no modelo teórico definido a priori, em que são discriminados os fatores associados à anemia em blocos hierarquizados, a primeira variável selecionada, incorporada ao modelo, foi

Tabela 1

Odds ratio (OR) da associação entre anemia e variáveis demográficas, sócio-econômicas e ambientais, em crianças e adolescentes de 7 a 14 anos de idade, da rede pública de ensino no Município de Salvador, Bahia, Brasil, 2007.

\begin{tabular}{|c|c|c|c|c|c|}
\hline Variável & $\mathrm{n}$ & Anêmicos (\%) & OR bruto & IC95\% & Valor de $p$ \\
\hline \multicolumn{6}{|l|}{ Sexo } \\
\hline Masculino & 515 & 24,32 & 1,00 & - & \\
\hline Feminino & 498 & 24,71 & 1,02 & $0,76-1,36$ & 0,886 \\
\hline \multicolumn{6}{|l|}{ Idade (anos) } \\
\hline $7-9,99$ & 274 & 20,27 & 1,00 & - & \\
\hline $10-14,99$ & 739 & 26,05 & 1,38 & $0,98-1,94$ & 0,061 \\
\hline \multicolumn{6}{|c|}{ Indicador das condições ambientais } \\
\hline \multicolumn{6}{|c|}{ e domiciliares de moradia * } \\
\hline$\leq 4$ adequado & 595 & 23,59 & 1,00 & - & \\
\hline$>4$ inadequado & 417 & 25,88 & 1,13 & $0,84-1,52$ & 0,412 \\
\hline \multicolumn{6}{|c|}{ 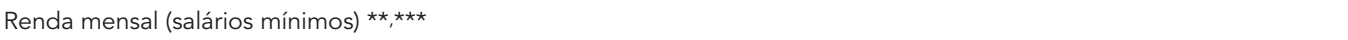 } \\
\hline$\geq 1$ & 734 & 22,73 & 1,00 & - & \\
\hline$<1$ & 273 & 28,85 & 1,37 & $1,00-1,90$ & 0,049 \\
\hline
\end{tabular}

* Informações faltantes ou inconsistência dos dados $=1$;

** Salário mínimo em dezembro de 2007: R\$380,00;

*** Informações faltantes ou inconsistência dos dados $=6$.

Tabela 2

Odds ratio (OR) da associação entre anemia e variáveis relativas ao consumo alimentar, em crianças e adolescentes de 7 a 14 anos de idade, da rede pública de ensino no Município de Salvador, Bahia, Brasil, 2007.

\begin{tabular}{|c|c|c|c|c|c|}
\hline Variável & $\mathrm{n}$ & Anêmicos (\%) & OR bruto & IC95\% & Valor de $p$ \\
\hline \multicolumn{6}{|c|}{ Adequação de calorias (\%) } \\
\hline$\geq 100$ & 371 & 22,80 & 1,00 & - & \\
\hline $50-99,9$ & 554 & 26,78 & 1,31 & $0,98-1,77$ & 0,067 \\
\hline$<50$ & 88 & 17,12 & 0,61 & $0,34-1,09$ & 0,099 \\
\hline \multicolumn{6}{|c|}{ Adequação de proteínas (\%) } \\
\hline$\geq 100$ & 895 & 24,45 & 1,00 & - & \\
\hline $50-99,9$ & 107 & 24,59 & 1,00 & $0,62-1,61$ & 0,984 \\
\hline$<50$ & 11 & 28,90 & 1,25 & $0,32-4,81$ & 0,741 \\
\hline \multicolumn{6}{|c|}{ Adequação de ferro total (\%) } \\
\hline$\geq 100$ & 351 & 19,96 & 1,00 & - & \\
\hline $50-99,9$ & 400 & 25,98 & 1,13 & $0,84-1,53$ & 0,388 \\
\hline$<50$ & 262 & 28,33 & 1,31 & $0,94-1,81$ & 0,102 \\
\hline \multicolumn{6}{|c|}{ Adequação de ferro biodisponível (\%) } \\
\hline$\geq 100$ & 288 & 19,44 & 1,00 & - & \\
\hline $50-99,9$ & 409 & 25,98 & 1,14 & $0,84-1,53$ & 0,380 \\
\hline$<50$ & 316 & 27,18 & 1,22 & $0,90-1,67$ & 0,191 \\
\hline
\end{tabular}


a renda familiar inferior a um salário mínimo ( $\mathrm{p}$ $=0,049$ ). Na segunda etapa da regressão logística foi introduzida, no modelo, além da renda familiar já selecionada na etapa anterior, aquelas variáveis pertencentes ao próximo nível hierárquico: número de habitantes por dormitório ( $\mathrm{p}=$ $0,122)$ e escolaridade materna $\left(p_{\text {dummy } 1}=0,218\right.$; $\mathrm{p}_{\mathrm{dummy} 2}=0,142$ ). Por não se manterem significativas, essas variáveis foram excluídas do modelo na etapa seguinte. Além da renda familiar já selecionada, integraram a etapa subseqüente as variáveis relacionadas à dimensão proximal de determinação da anemia: sexo $(p=0,886)$, idade $(\mathrm{p}=0,061)$ e estado antropométrico $\left(\mathrm{p}_{\text {dummyl }}=\right.$ 0,$124 ; p_{\text {dummy2 }}=0,106$ ). A variável sexo foi incorporada e mantida no modelo por ser conhecida como potencial confundidora da relação estudada. As variáveis idade e estado antropométrico não atenderam aos critérios estabelecidos previamente de permanência no modelo. Por último, em adição às variáveis renda familiar e sexo, foram introduzidas as relacionadas à adequação do consumo alimentar, as quais se associaram, significativamente, na análise univariada: adequação na ingestão de calorias ( $p_{\text {dummyl }}=0,067$; $\mathrm{p}_{\text {dummy2 }}=0,099$ ), a adequação na ingestão de ferro total $\left(\mathrm{p}_{\text {dummy } 1}=0,388 ; \mathrm{p}_{\text {dummy } 2}=0,102\right.$ ) e a adequação na ingestão de ferro biodisponível $\left(\mathrm{p}_{\text {dummy } 1}=0,380 ; \mathrm{p}_{\text {dummy } 2}=0,191\right)$.
Permaneceram, no modelo final, as variáveis: renda familiar, consumo de ferro biodisponível e sexo. Assim, indivíduos que consumiam uma dieta com o valor de ferro biodisponível entre $50 \%$ e $99,9 \%$ do recomendado apresentaram 1,57 vez (IC95\%: 1,07-2,29) mais chance de ter anemia do que aqueles que praticavam uma dieta com o valor de ferro biodisponível igual ou acima de $100 \%$ do recomendado. Essa chance elevou-se para 1,68 vez (IC95\%: 1,10-2,56) quando o consumo atingiu valores menores do que $50 \%$ do ferro biodisponível recomendado. Também os indivíduos oriundos de famílias com renda menor do que um salário mínimo apresentaram 1,42 vez mais chance de portar anemia do que aqueles oriundos de famílias que recebiam valores maiores do que esse patamar de renda (IC95\%: 1,03-1,96) (Tabela 4). O índice de bondade de ajustamento, avaliado pelo teste de Hosmer \& Lemeshow 18, indicou um bom modelo ( $\mathrm{p}=0,894)$.

Na Tabela 5, estão listados os grupos de alimentos, compostos pelos alimentos citados por pelo menos $10 \%$ das crianças e dos adolescentes. O almoço e o jantar foram as refeições que mais contribuíram com a quantidade de ferro biodisponível na dieta dos participantes $(0,41 \mathrm{mg}$ e $0,27 \mathrm{mg}$, respectivamente). Tais refeições agregaram, além dos alimentos fontes do mineral, aqueles potencializadores da absorção do ferro,

Odds ratio (OR) da associação entre anemia e variáveis relacionadas aos cuidados dispensados à criança e à antropometria, em crianças e adolescentes de 7 a 14 anos de idade, da rede pública de ensino no Município de Salvador, Bahia, Brasil, 2007.

\begin{tabular}{|c|c|c|c|c|c|}
\hline Variável & $\mathbf{n}$ & Anêmicos (\%) & OR bruto & IC95\% & Valor de $p$ \\
\hline \multicolumn{6}{|l|}{ Escolaridade materna * } \\
\hline 2o grau ou mais & 302 & 24,10 & 1,00 & - & \\
\hline 5ạ a 8ạ série & 364 & 27,39 & 1,25 & $0,92-1,69$ & 0,142 \\
\hline Até a 4ạ série & 324 & 22,24 & 0,81 & $0,59-1,12$ & 0,218 \\
\hline \multicolumn{6}{|c|}{ Número de crianças no domicílio com } \\
\hline \multicolumn{6}{|c|}{ menos do que cinco anos de idade ** } \\
\hline$\leq 2$ & 988 & 24,45 & 1,00 & - & \\
\hline$\geq 3$ & 24 & 27,89 & 1,19 & $0,48-2,94$ & 0,699 \\
\hline \multicolumn{6}{|c|}{ Número de habitantes por dormitório *** } \\
\hline$\leq 2$ & 477 & 22,27 & 1,00 & - & \\
\hline$\geq 3$ & 535 & 26,54 & 1,26 & $0,93-1,69$ & 0,122 \\
\hline \multicolumn{6}{|l|}{ Estado antropométrico } \\
\hline Eutrofia & 778 & 24,43 & 1,00 & - & \\
\hline Magreza & 133 & 29,93 & 1,37 & $0,91-2,06$ & 0,124 \\
\hline Sobrepeso/Obesidade & 102 & 17,97 & 0,64 & $0,38-1,09$ & 0,106 \\
\hline
\end{tabular}

* Informações faltantes ou inconsistência dos dados = 23;

** Informações faltantes ou inconsistência dos dados $=1$;

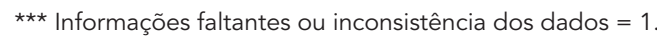


Odds ratio (OR) ajustado * para avaliação dos fatores associados à anemia em crianças e adolescentes de 7 a 14 anos de idade, da rede pública de ensino no Município de Salvador, Bahia, Brasil, 2007.

\begin{tabular}{|c|c|c|c|}
\hline Variável & OR ajustado & IC95\% & Valor de $\mathrm{p}$ \\
\hline \multicolumn{4}{|c|}{ Determinantes estruturais ou básicos } \\
\hline \multicolumn{4}{|c|}{ 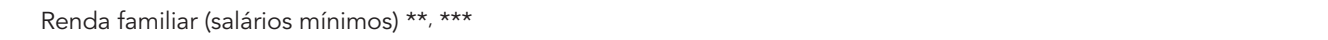 } \\
\hline$\geq 1$ & 1,00 & - & \\
\hline$<1$ & 1,42 & $1,03-1,96$ & 0,035 \\
\hline \multicolumn{4}{|c|}{ Determinantes proximais } \\
\hline \multicolumn{4}{|c|}{ Adequação de ferro biodisponível (\%) } \\
\hline$\geq 100$ & 1,00 & - & \\
\hline $50-99,9$ & 1,57 & $1,07-2,29$ & 0,020 \\
\hline$<50$ & 1,68 & $1,10-2,56$ & 0,016 \\
\hline
\end{tabular}

a exemplo das carnes, cujo consumo médio foi de 43,45g e 40,86g, respectivamente, no almoço e no jantar. É inexpressiva a quantidade de vitamina C - outro potente favorecedor da absorção do ferro não heme - oferecida no almoço $(4,22 \mathrm{~g})$ e no jantar $(4,7 \mathrm{~g})$. No almoço, as leguminosas, basicamente os feijões, contribuíram com 2,23g/ dia de ferro. As verduras e as frutas não compõem o cardápio da grande maioria dos indivíduos investigados.

\section{Discussão}

Os resultados deste estudo indicam que a anemia é considerada um problema de saúde pública moderado em população de crianças e adolescentes da rede pública de Ensino Fundamental da cidade de Salvador 2 . A prevalência de anemia encontrada no presente estudo $(24,5 \%)$ é similar à observada por Santos et al. 19, em favelas da zona sul de São Paulo $(24,4 \%)$ e por Tsuyuoka et al. 20 na capital de Sergipe $(26,7 \%)$. Trata-se de uma taxa de prevalência mais elevada do que a revelada em outro estudo realizado em Maceió, capital do Estado de Alagoas por Santos et al. 21 $(9,9 \%)$, e também da investigação desenvolvida por Frutuoso et al. 22 no Município de São Paulo $(7,7 \%)$, por Moura et al. 23 no Município de Campinas, São Paulo (19,3\%), por Fujimori et al. 24 no Município de Taboão da Serra, São Paulo (17,6\%) e por Heijblom \& Santos 25 na Regional Norte de Saúde de Brasília (11,9\%). Entretanto, taxas de prevalências maiores do que a observada no estudo em pauta foram registradas por Brito et al. 8, em Jequié, município do Estado da Bahia
(32,2\%), por Uchimura et al. 26 em Maringá, Estado do Paraná (31,7\%), por Ferreira et al. 27, em São Lourenço da Mata, Estado de Pernambuco (43,1\%), por Miglioranza et al. 28, em Londrina no Estado do Paraná (41,3\%), por Mariath et al. 29 em Camburiú, Santa Catarina (31,2\%), e por Stefanini et al. 30 no Município de Osasco, em São Paulo (51\%). Os critérios adotados para o diagnóstico de anemia em cada estudo podem justificar a variação observada. Contudo, independentemente da metodologia, destacam-se as elevadas prevalências desta deficiência nutricional nesta fase da vida.

Os resultados deste estudo revelaram que as variáveis dos contextos da dimensão estrutural e da dimensão proximal se associaram à anemia entre as crianças e os adolescentes pesquisados. Nesse sentido, verifica-se que o patamar de renda menor do que um salário mínimo imprimia maior chance de o indivíduo ser portador da anemia, quando comparado com o nível de renda mais elevado $(\mathrm{OR}=1,42$; IC95\%: 1,03-1,96; $\mathrm{p}=$ $0,035)$. Esse resultado assemelha-se àqueles encontrados por Brito et al. ${ }^{8}$, na Bahia; Neumann et al. 31 , em Criciúma, Santa Catarina; e Silva et al. 32, em Porto Alegre, Rio Grande do Sul. Para esses autores, embora indivíduos de qualquer estrato não se apresentem imunes à anemia, as famílias de menor nível sócio-econômico estão submetidas a um maior risco de desenvolver a doença, e sua prevalência tende a ser menor nos estratos de melhor nível sócio-econômico. A relevância do nível de renda na determinação da anemia parece decorrer do amplo efeito que ele exerce sobre a quantidade e a qualidade dos alimentos - em especial do consumo de alimentos 
Contribuição dos diferentes alimentos para o fornecimento de ferro, de acordo com as refeições realizadas, a crianças e adolescentes de 7 a 14 anos de idade, da rede pública de ensino no Município de Salvador, Bahia, Brasil, 2007.

\begin{tabular}{|c|c|c|c|}
\hline Refeição & Média (g) & Erro padrão (g) & Ferro total (mg) \\
\hline \multicolumn{4}{|l|}{ Café da manhã } \\
\hline Leite + derivados * & 127,30 & 3,78 & 0,10 \\
\hline Cereais + derivados ** & 90,21 & 1,86 & 1,91 \\
\hline 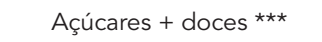 & 17,85 & 0,51 & 0,01 \\
\hline Óleo + gorduras \# & 12,98 & 0,83 & 0,01 \\
\hline \multicolumn{4}{|c|}{ Ferro biodisponível =0,066mg } \\
\hline \multicolumn{4}{|c|}{ Lanche } \\
\hline Bebidas \#\# & 188,04 & 9,85 & 0,56 \\
\hline Leite + derivados \#\#\# & 113,78 & 7,32 & 0,32 \\
\hline Cereais + derivados $\S$ & 91,30 & 4,16 & 0,97 \\
\hline Açúcares + doces §§ & 22,94 & 1,61 & 0,06 \\
\hline \multicolumn{4}{|c|}{ Ferro biodisponível =0,150mg } \\
\hline \multicolumn{4}{|c|}{ Almoço } \\
\hline Cereais + derivados §§§ & 92,16 & 2,02 & 1,20 \\
\hline Leguminosas $\dagger$ & 212,29 & 4,72 & 2,23 \\
\hline Carne + derivados $\dagger^{\dagger}$ & 46,91 & 1,51 & 1,44 \\
\hline \multicolumn{4}{|c|}{ Ferro biodisponível = 0,410mg } \\
\hline \multicolumn{4}{|c|}{ Lanche } \\
\hline Bebidas t+† & 208,91 & 9,86 & 0,44 \\
\hline Cereais + derivados $\ddagger$ & 88,04 & 4,08 & 0,56 \\
\hline Açúcares + doces 㧊 & 29,89 & 1,92 & 0,08 \\
\hline \multicolumn{4}{|c|}{ Ferro biodisponível =0,086mg } \\
\hline \multicolumn{4}{|l|}{ Jantar } \\
\hline Leite + derivados $\ddagger \ddagger \ddagger$ & 117,49 & 3,61 & 0,11 \\
\hline Cereais + derivados \& & 95,66 & 2,04 & 1,66 \\
\hline Carne + derivados \&\& & 48,07 & 2,71 & 1,21 \\
\hline Açúcares + doces \&\&\& & 19,71 & 0,75 & 0,02 \\
\hline Óleo + gorduras q & 14,12 & 0,66 & 0,02 \\
\hline \multicolumn{4}{|c|}{ Ferro biodisponível = 0,271 mg } \\
\hline \multicolumn{4}{|c|}{ Absorção de ferro biodisponível total no dia $=0,984 \mathrm{mg}$} \\
\hline \multicolumn{4}{|l|}{ * $88,81 \%$ de leite; } \\
\hline \multicolumn{4}{|c|}{ ** $74,66 \%$ de pão e $11,83 \%$ de biscoito; } \\
\hline \multicolumn{4}{|c|}{ 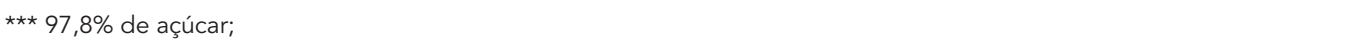 } \\
\hline \multicolumn{4}{|l|}{ \# 98,57\% de manteiga; } \\
\hline \multicolumn{4}{|c|}{ \#\# 8,27\% de achocolatado, $20 \%$ de refrigerante e $64,14 \%$ de suco; } \\
\hline \multicolumn{4}{|c|}{ \#\#\# 64,9\% de leite e 14,57\% de bebida láctea; } \\
\hline \multicolumn{4}{|c|}{$\S 27,98 \%$ de biscoito, $27,72 \%$ de pão e $10,36 \%$ de salgadinho; } \\
\hline \multicolumn{4}{|c|}{$\S \S 53,2 \%$ de açúcar e $46,8 \%$ de doces; } \\
\hline \multicolumn{4}{|c|}{$\S \S \S 51,7 \%$ de arroz, $29,2 \%$ de farinha de mandioca e $13,2 \%$ de macarrão; } \\
\hline \multicolumn{4}{|c|}{ † 98,9\% de feijão; } \\
\hline \multicolumn{4}{|c|}{ t† $52,16 \%$ de carne bovina, $21,93 \%$ de frango e $18,68 \%$ de embutidos; } \\
\hline t†† $65,03 \%$ de suco e $27,81 \%$ & & & \\
\hline$\ddagger 41,33 \%$ de biscoito, $14,49 \%$ & algadinho; & & \\
\hline 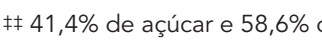 & & & \\
\hline 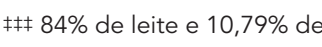 & & & \\
\hline$\& 50,15 \%$ de pão, $13,5 \%$ de & & & \\
\hline$\& \& 42,1 \%$ de carne, $33,47 \%$ d & de frango; & & \\
\hline \&\&\& 89,6\% de açúcar; & & & \\
\hline १ $86,17 \%$ de manteiga. & & & \\
\hline
\end{tabular}


fontes de ferro heme, como as carnes -, o acesso aos serviços de saúde e a qualidade da moradia e saneamento. Segundo Szarfarc et al. ${ }^{33}$, a escassez e a má distribuição desses serviços determinam práticas alimentares inadequadas, infestações parasitárias freqüentes, que estão, por fim, associadas ao baixo poder aquisitivo da população.

Os resultados encontrados no estudo em questão mostraram que a anemia foi significantemente associada ao consumo alimentar inadequado de ferro biodisponível. Observou-se que a adequação de consumo entre 50\% e 99,9\% de ferro biodisponível imprimiu chance 1,57 vez (IC95\%: 1,07-2,29; p = 0,020) maior de a criança ser portadora da anemia, quando comparada com a chance impressa por níveis de adequação maiores do que $100 \%$. Essa chance elevou-se para 1,68 vez (IC95\%: 1,10-2,56; $\mathrm{p}=0,016$ ), quando o consumo atingiu valores menores do que $50 \%$ do ferro biodisponível recomendado. Esses resultados, similares àqueles encontrados por Brito et al. 8, fornecem evidências de que, a baixa biodisponibilidade do ferro dietético constitui um fator crítico na ocorrência da anemia.

A biodisponibilidade do ferro alimentar depende, dentre outros fatores, da forma como esse nutriente está veiculado na refeição; há que registrar o triplo papel das carnes no aproveitamento do ferro; elas são fontes do mineral (cerca de $4 \mathrm{mg} / 100$ do total); contêm ferro hemínico (hemoglobina e mioglobina - cerca de $40 \%$ do total) com características ímpares no que se refere à absorção; e potencializam a absorção do po$o l$ de ferro não hemínico presente nas refeições. Esse papel de facilitador da absorção do ferro não heme ainda não está totalmente esclarecido; acredita-se que produtos da digestão protéica (aminoácidos e polipeptídios) podem formar quelatos solúveis com o ferro não heme 34 . Alguns autores ainda sugerem que as carnes favorecem a neutralização de fatores que poderiam interferir na liberação do ferro do alimento e no seu transporte para a célula 34,35 .

No presente estudo, a prática alimentar constituiu um fator crítico na determinação da anemia. Os dados demonstraram que o feijão com a farinha, acompanhados por uma pequena porção de carne, foram os principais itens alimentares identificados nas refeições mais importantes do dia da grande maioria das crianças e dos adolescentes. Contudo, a ínfima porção de carne, em torno de $47 \mathrm{~g}$, ganha relevância na medida em que a maior parte do ferro dietético disponível nas dietas é do tipo não heme, proveniente dos alimentos de origem vegetal. Além disso, observou-se que um contingente apreciável de indivíduos não consumia alimentos fontes de vitamina
C - menos que $10 \%$-, outro potente favorecedor da absorção do ferro não heme. O ácido ascórbico atua de duas formas: mantendo o ferro dos alimentos no estado ferroso (mais solúvel), por ser um agente redutor, e mantendo-o na forma de quelato ferro-ascorbato, que é solúvel 36 . Segundo De Mayer et al. 37 o consumo de ácido ascórbico junto às refeições torna-se uma medida eficiente para o aumento da absorção do ferro e a diminuição da anemia. Assim, os resultados deste estudo demonstraram que, além de evidenciarem baixo consumo de alimentos favorecedores da absorção de ferro não heme, os participantes consomem pequenas quantidades de alimentos que contêm ferro heme.

Resultados das poucas investigações que avaliam a relação entre o padrão de consumo alimentar e a ocorrência da anemia corroboram com os dados do presente estudo, que indicam a inadequação da prática alimentar como fator importante na ocorrência dessa deficiência. $\mathrm{O}$ reduzido consumo de alimentos que contêm ferro heme, além do baixo consumo de alimentos favorecedores da absorção de ferro não heme, como as frutas e as hortaliças, indicam a má qualidade da dieta consumida pelas crianças e pelos adolescentes 38 . Alguns estudos têm mostrado que são características da alimentação dos jovens na atualidade a substituição das refeições principais pelos fast foods 38,39 . Por apresentarem alta quantidade de energia e baixa quantidade de ferro, cálcio, vitamina A e fibras, o impacto nutricional causado por esse tipo de alimentação pode causar sérios distúrbios nutricionais a essa população.

O caráter probabilístico e a implementação bem sucedida dos procedimentos empregados para a seleção amostral demonstram que os resultados do presente estudo podem ser estendidos para o conjunto de crianças e adolescentes, com idade entre 7 e 14 anos de idade, matriculados na rede pública de ensino do Município de Salvador.

É importante salientar que a principal limitação desta investigação está no fato de se tratar de um estudo transversal, o que afeta a interpretação dos resultados, visto que nesse tipo de estudo não é possível estabelecer relações causais por não evidenciarem uma seqüência temporal entre a exposição ao fator e o subseqüente desenvolvimento da doença. Por conseguinte, o desenho adotado permite apenas observar associações entre os eventos estudados, não sendo possível demonstrar uma relação de causa e efeito entre tais eventos.

O método recordatório de 24 horas, utilizado neste estudo para investigar o consumo alimentar, também apresenta algumas limitações. 
Apesar de ser um método rápido, de custo relativamente baixo e de fácil aplicação, o êxito na sua utilização depende da memória do entrevistado e requer treinamento do pesquisador para a obtenção de estimativas acuradas das porções consumidas. No entanto, esse método fornece informações confiáveis da estimativa do consumo médio das populações, inclusive quando aplicado uma única vez, quando os pressupostos metodológicos são observados e os recursos analíticos, adequados 40. Assim, os estudos de consumo alimentar podem fornecer, precocemente, importantes informações sobre os riscos de deficiência nutricional, antes que a forma clínica se instale 40.

\section{Resumo}

Trata-se de um estudo para avaliar os fatores associados à anemia. Participaram deste estudo 1.013 indivíduos com idade entre 7 e 14 anos, matriculados na rede pública de ensino em Salvador, Bahia, Brasil. Os participantes foram submetidos a exame de sangue para dosagem de hemoglobina, exame antropométrico e obtiveram-se informações sobre consumo alimentar e condições sócio-econômicas, domiciliares e ambientais da família, além do cuidado materno dispensado à criança. Foi detectada uma prevalência de $24,5 \%$ de anêmicos entre os participantes, com valor médio dos níveis de hemoglobina de 12,68g/dL, concentração mínima de $7,7 \mathrm{~g} / \mathrm{dL}$ e máxima de $16,8 \mathrm{~g} / \mathrm{dL}$. A anemia foi significativamente associada à inadequação crescente de consumo de ferro biodisponivel $\left(O R_{\text {adequač̃o entre } 50 \% \text { a 99,9\% }}=1,57\right.$; IC95\%: 1,07-2,29; $p=0,020$ e OR adequaç̃̃o < 50\% $=1,68 ;$ IC95\%: 1,10-2,56; $p=0,016)$ e à renda familiar menor do que 1 salário mínimo $(O R=1,42$; IC95\%: 1,03-1,96; $p=0,035)$. A prevalência de anemia em crianças e adolescentes é elevada, especialmente em indivíduos mais pobres e com baixo consumo de ferro biodisponível.

Anemia; Consumo Alimentar; Criança; Adolescente
Concluindo, a prevalência de anemia no grupo de crianças e adolescentes estudado é moderada, especialmente entre aqueles mais pobres $\mathrm{e}$ cujas dietas têm baixo teor de ferro biodisponível. Os estudos apontam para o acelerado crescimento da prevalência de anemia no país, nas últimas décadas. Neste contexto, o país vem adotando políticas públicas a fim de controlar a ocorrência da anemia, a exemplo de: suplementação medicamentosa de ferro em gestantes e crianças dos 6 a 18 meses de idade, além de preconizar estratégias de educação nutricional. Entretanto, num curto prazo, é necessário implementar outras medidas, como forma de benefício dirigido sobretudo aos grupos de crianças em idade escolar e adolescentes, a exemplo da inclusão de alimentos fontes de ferro de alta biodisponibilidade na alimentação escolar.

\section{Colaboradores}

C. Q. Borges e R. C. R. Silva foram responsáveis pela concepção do estudo, levantamento bibliográfico, coleta e análise dos dados, interpretação dos resultados e redação do manuscrito. A. M. O. Assis participou da concepção do estudo, interpretação dos resultados e redação do manuscrito. R. L. Fiaccone, E. J. Pinto e S. M. C. Pinheiro contribuíram na análise dos dados, interpretação dos resultados e redação do manuscrito.

\section{Agradecimentos}

À Fundação de Amparo à Pesquisa do Estado da Bahia (FAPESB), pelo financiamento do projeto Situação $\mathrm{Nu}$ tricional, Hábitos Alimentares e Desenvolvimento Cognitivo de Estudantes do Ensino Fundamental das Escolas Públicas de Salvador/BA (processo no ${ }^{\circ}$. 1431040053551) e deste estudo (processo $\mathrm{n}^{\circ}$. 195712163383). À Secretaria Municipal de Saúde do Salvador, Secretaria Municipal de Educação e Cultura do Salvador e Secretaria Estadual de Educação da Bahia. 


\section{Referências}

1. Stoltzfus RJ. Iron-deficiency anemia: reexamining the nature and magnitude of the public health problem. Summary: implications for research and programs. J Nutr 2001; 131(2S-2):697S-700S.

2. World Health Organization. Iron deficiency anaemia: assessment, prevention and control: a guide for programme managers. Geneva: World Health Organization; 2001.

3. Chwang LC, Soemantri AG, Pollitt E. Iron supplementation and physical growth of rural Indonesian children. Am J Clin Nutr 1988; 47:496-501.

4. Pollitt E. Early iron deficiency anemia and later mental retardation. Am J Clin Nutr 1999; 69:4-5.

5. Lozoff B, De Andraca I, Castillo M, Smith JB, Walter T, Pino P. Behavioral and developmental effects of preventing iron-deficiency anemia in healthy fullterm infants. Pediatrics 2003; 112:846-54.

6. Lozoff B, Jimenez E, Hagen J, Mollen E, Wolf AW. Poorer behavioral and developmental outcome more than 10 years after treatment for iron deficiency in infancy. Pediatrics 2000; 105:E51.

7. Finch CA, Cook JD. Iron deficiency. Am J Clin Nutr 1984; 39:471-7.

8. Brito LL, Barreto ML, Silva RCR, Assis AMO, Reis MG, Parraga I, et al. Fatores de risco para anemia por deficiência de ferro em crianças e adolescentes parasitados por helmintos intestinais. Rev Panam Salud Pública 2003; 14:422-31.

9. Mills AF, Meadows N. Screening for anaemia: evaluation of a haemoglobinometer. Arch Dis Child 1989; 64:1468-71.

10. Unite for Children/United Nations University/ World Health Organization/Micronutrient Initiative. Preventing iron deficiency in women and children: technical consensus on key issues and resources for programme advocacy, planning and implementation. New York: Unite for Children; 1998.

11. Food and Agriculture Organization of the United Nations/World Health Organization/United Nations University. Human energy requirements: report of a joint expert consultation. Rome: Food and Agriculture Organization of the United Nations/World Health Organization/United Nations University; 2001

12. Food and Agriculture Organization of the United Nations/World Health Organization/United Nations University. Protein and amino acid requirements in human nutrition: Report of a joint expert consultation. Geneva: Food and Agriculture Organization of the United Nations/World Health Organization/United Nations University; 2007.

13. Monsen ER, Balintfy JL. Calculating dietary iron bioavailability: refinement and computerization. J Am Diet Assoc 1982; 80:307-11.

14. Lohman TG, Roche AF, Martorell R. Anthropometric standardization reference manual. Champaign: Human Kinetics Books; 1988.

15. WHO Multicentre Growth Reference Study Group. WHO Child Growth Standards: length/height-forage, weight-for-age, weight-for-length, weight-forheight and body mass index-for-age: methods and development. Geneva: World Health Organization; 2006.
16. World Health Organization. Physical status: the use and interpretation of anthropometry. Report of a WHO Expert Committee. Geneva: World Health Organization; 1995. (Technical Report Series, 854).

17. Issler RM, Giugliani ER. Identificação de grupos mais vulneráveis à desnutrição infantil pela medição do nível de pobreza. J Pediatr (Rio J) 1997; 73:101-5.

18. Hosmer DW, Lemeshow S. Applied logistic regression. New York: John Wiley and Sons; 1989.

19. Santos EB, Amancio OMS, Oliva CAG. Estado nutricional, ferro, cobre e zinco em escolares de favela da cidade de São Paulo. Rev Assoc Méd Bras 2007; 53:323-8.

20. Tsuyuoka R, Bailey JW, Guimarães AMd'AN, Gurgel RQ, Cuevas LE. Anemia and intestinal parasitic infections in primary school students in Aracaju, Sergipe, Brazil. Cad Saúde Pública 1999; 15: 413-21.

21. Santos CD, Santos LMP, Figueiroa JN, Marroquim PMG, Oliveira MAA. Anemia em escolares da primeira série do ensino fundamental da rede pública de Maceió, Alagoas, Brasil. Cad Saúde Pública; 18:1757-63.

22. Frutuoso MFP, Vigantzky VA, Gambardelella AMD. Níveis séricos de hemoglobina em adolescentes segundo estágio de maturação sexual. Rev Nutr 2003; 16:155-62.

23. Moura EC, Santos AM, Pacheco CE. Anemia ferropriva em escolares de Campinas, São Paulo: prevalência, sensibilidade e especificidade de testes laboratoriais. Rev Bras Saúde Matern Infant 2001; 1:123-7.

24. Fujimori E, Szarfarc S, Oliveira IMV. Prevalência de anemia e deficiência de ferro em adolescentes do sexo feminino: Taboão da Serra, SP, Brasil. Rev Latinoam Enferm 1996; 4:49-63.

25. Heijblom GS, Santos LMP. Anemia ferropriva em escolares da primeira série do ensino fundamental da rede pública de educação de uma região de Brasília, DF. Revista Bras Epidemiol 2007; 10 258-66.

26. Uchimura TT, Szarfarc SC, Latorre MRDO, Uchimura NS, Souza SB. Anemia e peso ao nascer. Rev Saúde Pública 2003; 37:397-403.

27. Ferreira MR, Souza W, Perez EP, Lapa T, Carvalho AB, Furtado A, et al. Intestinal Helminthiasis and Anaemia in Youngsters from Matriz da Luz, District of São Lourenço da Mata, State of Pernambuco, Brazil. Mem Inst Oswaldo Cruz 1998; 93: 289-93.

28. Miglioranza LHS, Matsuo T, Caballero-Córdoba GM, Dichi JB, Cyrino ES, Oliveira IBN, et al. Prevalência de anemia em crianças e adolescentes de unidades educacionais na periferia de Londrina, PR. Rev Nutr 2002; 15:149-53.

29. Mariath AB, Henn R, Matos CH, Lacerda LLV, Grilo LP. Prevalência de anemia e níveis séricos de hemoglobina em adolescentes segundo estágio de maturidade sexual. Rev Bras Epidemiol 2006; 9:454-61. 
30. Stefanini MLR, Colli C, Lerner BR, Lei DLM, Chaves SP, Di Pietro MS, et al. Anemia e desnutrição em escolares da rede pública do município de Osasco, São Paulo, Brasil. Cad Saúde Pública 1995; 11:439-47.

31. Neuman NA, Tanaka OY, Szarfarc SC, Guimarães PR, Victora CG. Prevalência e fatores de risco para anemia no Sul do Brasil. Rev Saúde Pública 2000; 34:56-63.

32. Silva LSM, Giugliani ERJ, Aerts DRGC. Prevalência e determinantes de anemia em crianças de Porto Alegre, RS, Brasil. Rev Saúde Pública 2001; 35: 66-73.

33. Szarfarc SC, Stefanini MLR, Lerner BR. Anemia nutricional no Brasil. Cadernos de Nutrição 1995; 9:5-24.

34. Storcksdieck S, Bonsmann G, Hurrell RF. Ironbinding properties, amino acid composition, and structure of muscle tissue peptides from in vitro digestion of different meat sources. J Food Sci 2007; 72:S019-29.

35. Lynch SR, Hurrell RF, Dassenko SA, Cook JD. The effect of dietary proteins on iron bioavailability in man. Adv Exp Med Biol 1989; 249:117-32.
36. Cook JD, Reddy MB. Effect of ascorbic acid intake on nonheme-iron absorption from a complete diet. Am J Clin Nutr 2001; 73:93-8.

37. DeMaeyer EM, Dallman P, Guerney JM, Hallberg L, Sood SK, Srikantia SG. Preventing and controlling iron deficiency anaemia through primary health care: a guide for health administrators and programme managers. Geneva: World Health Organization; 1989.

38. Matos CH, Grillo LP, Henn R, Germani AC. Anemia ferropriva em adolescentes de escolas municipais de Balneário Camboriú-SC. Nutrição em Pauta 2003; 60:48-53.

39. Andersen LF, Nes M, Sandstad B, Bjorneboe GE, Drevon CA. Dietary intake among Norwegian adolescents. Eur J Clin Nutr 1995; 49:555-64.

40. Willett W. Nutritional epidemiology. New York: Oxford University Press; 1990.

Recebido em 18/Mar/2008

Versão final reapresentada em 15/Set/2008 Aprovado em 10/Out/2008 